\title{
Treat Other HIV-Associated Complications
}

National Cancer Institute

\section{Source}

National Cancer Institute. Treat Other HIV-Associated Complications. NCI Thesaurus.

Code C19091.

Improved understanding of the pathogenesis of these disorders can be applied to the development and evaluation of therapies for the treatment and prevention of serious HIV-associated complications, especially wasting syndrome and growth failure, as well as other disorders including hematologic, dermatologic, renal, metabolic, pulmonary, cardiac, endocrinologic, psychiatric, and oral manifestations. 